\title{
Pathogen inactivation: prospects and pitfalls
}

Keywords: HIV, HBV, HCV, blood transfusion, pathogen inactivation, UVA

\section{Introduction}

While the spread of viral diseases like HIV, HBV, HCV via blood transfusion have been controlled to a large extent but the threats from emerging pathogens and the bacterial contamination of platelets concentrates (PC) remains a major threat with serious clinical consequences. In contrast to the well-established pathogen inactivation strategies for fresh frozen plasma using the solventdetergent procedure or methylene blue and visible light, the bench -to-bedside translation of novel pathogen inactivation technologies for cell-containing blood components such as platelets and red blood cells are still underway. ${ }^{1}$

Pathogen inactivation (PI) systems have been developed that have proven to be effective against numerous bacteria, viruses, and parasites. Two main systems have emerged to treat platelet concentrates: treatment with psoralen ultraviolet A (UVA) light and treatment with riboflavin (vitamin B2) plus ultraviolet B (UVB) light, both targeting the nucleic acids of pathogens. The ability of the Mirasol PRT system to inactivate both pathogens and white blood cells has been previously described.1The technology uses a combination of riboflavin and UV light to induce irreversible lesions in the nucleic acids of pathogens and white blood cells (WBCs) to inhibit replication and function. ${ }^{2}$

Hence, PI techniques can be considered as a "Paradigm shift" in ensuring safer blood transfusion as PI uses a variety of physical, chemical, or photochemical methods to remove or inactivate that blood-borne pathogens, such as viruses, bacteria, and parasites in blood components or products. These PI methods include but not limit to solvent /detergent (S/D), nanofiltration and photochemical inactivation such as using Methylene Blue (MB), psoralens, or riboflavin.

Nowadays, research on PI technology for blood components (plasma and platelet) has made a great progress. Several inactivation methods including MB, Psoralens and Riboflavin can be chosen. These methods are targeting viral nucleic acids (NA) through photochemical inactivation. Methylene Blue (MB) is a phenothiazinium dye with a natural affinity for NA. Once exposed to visible light (620-670nm), $\mathrm{MB}$ can release reactive oxygen species (mostly singlet oxygen) by photodynamic reaction to induce the guanine-specific cleavage of viral NA, resulting in virus activation. MB is effective in inactivating envelop virus. Although some allergic adverse events have been occasionally reported, $\mathrm{MB}$ has been used to inactivate single plasma units in 18 countries with minimal toxicity, confirming the long-term safety of MB - treated plasma. ${ }^{3}$

Amotosalen (S-59), known as photosensitizers, is a synthetic psoralen obtained from numerous plants. Through a three-step of intercalation, monoadduct and cross-links (diadducts) chemical reactions between single-or double-stranded DNA or RNA, UVA-light mediated amotosalen inhibits nucleic acids replication, transcription and repair mechanism as well. Intercept plasma (Cerus, Concord, CA), as a successfully developed commercial product of Amotosalen/
Volume 5 Issue 6 - 2017

\author{
Subhashish Das \\ Professor of Pathology, Sri Devaraj Urs Medical College, India
}

Correspondence: Subhashish Das, Department of Pathology, Sri Devaraj Urs Medical College, Tamaka, Kolar-563101, Karnataka, India, Tel 08I52220528/222487, Mob 094808498I8, Email daspathology@gmail.com

Received: October 23, 2017 | Published: November 24, 2017

UVA, has been used for nearly a decade in more than 20 countries. This technology has been proved to be effective to inactivate a broad spectrum of viruses, bacteria and parasites as well as leukocytes contained in the blood products and considered to be safe without any unusual adverse effects or toxicity events. ${ }^{4}$

Riboflavin (vitamin B2)-based compounds work through UVB light (265-37nm) illumination. This photodynamic reaction generates single oxygen, which is responsible for photo-oxidation of guanine bases and results in strand breaks and fragmentations, thus damaging nucleic acids (DNA or RNA) irreversibly. Riboflavin, the so called "GRAS" (Generally Regarded as Safe) products by the Federal Drug Administration (FDA), due to its wide existence in a variety of natural foods and human blood, neither riboflavin nor its metabolic products need to be removed after this PI treatment. Many studies have demonstrated the effectiveness of this technology in inactivating a wide range of pathogens (bacteria, viruses and protozoa etc). Mirasol (Terumo BCT, USA), based on Riboflavin/UVB PI technology, has been used in a number of blood centers in many countries. ${ }^{5}$

\section{The advantages of PI include}

The advantages of PI includes (a) PI methods are particularly efficient to prevent transfusion-related bacterial infections (b) PI methods of PC globally reduce the risk of transfusion transmitted diseases and replace $\gamma$-irradiation for the prevention of GvHD. (c) Hemostatic efficacy of PI-treated PC appears to be maintained, although the CCI is lower when compared to untreated PC.

In a study conducted by Castrillo et al., ${ }^{2}$ the PC in PAS treated with the Mirasol system demonstrated minimal loss of platelet quality parameters. A minimal reduction in swirling was noted on the $7^{\text {th }}$ day of storage, indicating that PC morphology was preserved in all units.

In vitro studies have shown that PI-treated PC has a raised activation level with increased metabolic parameters which needs to be further verified on a larger scale with extensive documentation. Activated fibrinogen receptor expression appears to be increased after PI, perhaps through a direct effect of PI on this integrin. These data relate mainly to the amotosalen/UVA technique and, to lesser extent, to the riboflavin/UV method. ${ }^{5}$

PI of blood components is a solid next step to eliminate the threat of transfusion transmittable disease in the blood supply. However, a well-balanced interplay of different aspects is needed to successfully 
implement these worldwide. One of the most important criteria to ascertain include the cost- economics and the feasibility for wider application particularly in high demands and resource constrained setup as seen in developing countries. However, recent analyses have demonstrated that PRT-treated blood products may actually reduce the overall health care costs and the duration of hospital stay associated with post-transfusion care for some patients. Due to the ability of the Mirasol PRT treatment to inactivate all remaining leukocytes in a product that mediate GvHD, it can be used as an alternative to $\gamma$-irradiation thereby saving huge medical cost and ensuring increased patient comfort and satisfaction. ${ }^{6}$

In addition, the Mirasol PRT system, which is considered to be the gold standard to inactivate residual leukocytes in blood products, has been shown to prevent accumulation and secretion of most WBC -associated cytokines, with the potential to prevent leukocytesmediated immunologic reactions in recipients. ${ }^{6}$

The Mirasol system is easy to use and does not require special training to operate the equipment. This technology can eliminate most of the residual risk of bacteria and also reduces the risk associated with a long list of transfusion-transmitted pathogens for which the blood supply is not screened.

More studies are required to fully understand the various mechanisms involved for PI and hence PI remains a challenge in the near future, which in turn, call's for more concerted efforts for sustained and long term clinical benefits.

\section{Acknowledgements}

None.

\section{Conflict of interest}

The author declares no conflict of interest.

\section{References}

1. Mintz PD. Cesium cessation? An advantage of pathogen reduction treatments. Transfusion. 2011;51(7):1369-1376.

2. Castrillo SM, Schneider V, Gathof BS. Functional characteristics of apheresis-derived platelets treated with ultraviolet light combined with either amotosalen-HCI(S-59) or riboflavin (vitamin B2) for pathogenreduction. Vox Sang. 2009;97(1):26-33.

3. Reikvam H, Marschner S, Apelseth TO, et al. The Mirasol Pathogen Reduction Technology system and quality of platelets stored in platelet additive solution. Blood Transfus. 2010;8(3):186-192.

4. Webert KE, Cserti CM, Hannom J, et al. Proceedings of a consensus conference: pathogen inactivation making decision about new technologies. Transfus Med Rev. 2008;22(1):1-34.

5. Marschner S, Fast LD, Baldwin WM, et al. White blood cell inactivation after treatment with riboflavin and ultraviolet light. Transfusion. 2010;50(11):2489-2498.

6. Solheim BG. Pathogen reduction of blood components. Transfus Apher Sci. 2008;39(1):75-82. 